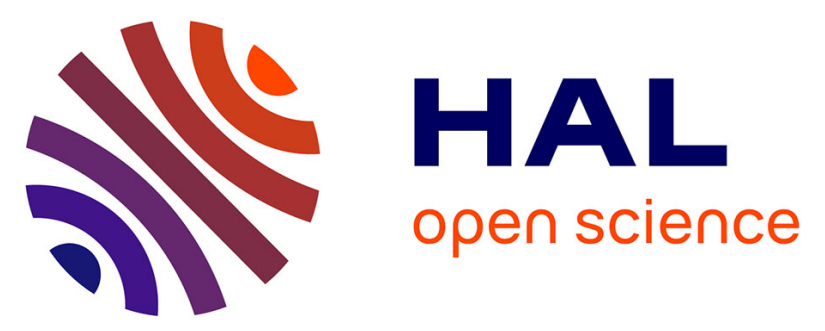

\title{
Noncircularity exploitation in signal processing overview and application to radar
}

\author{
F. Barbaresco, Pascal Chevalier
}

\section{To cite this version:}

F. Barbaresco, Pascal Chevalier. Noncircularity exploitation in signal processing overview and application to radar. IET Waveform Diversity \& Digital Radar Conference 2008. Day 1: Waveform Diversity \& Design, Dec 2008, London, United Kingdom. pp.3-3, 10.1049/ic.2008.0743 . hal-02469905

\section{HAL Id: hal-02469905 https://hal.science/hal-02469905}

Submitted on 6 Feb 2020

HAL is a multi-disciplinary open access archive for the deposit and dissemination of scientific research documents, whether they are published or not. The documents may come from teaching and research institutions in France or abroad, or from public or private research centers.
L'archive ouverte pluridisciplinaire HAL, est destinée au dépôt et à la diffusion de documents scientifiques de niveau recherche, publiés ou non, émanant des établissements d'enseignement et de recherche français ou étrangers, des laboratoires publics ou privés. 


\title{
Noncircularity exploitation in Signal Processing Overview and Application to Radar
}

\author{
Frederic Barbaresco (1) and Pascal Chevalier (2) \\ (1) THALES AIR SYSTEMS, SR/STI, Hameau de Roussigny, F-91470, Limours, France \\ (2) THALES COMMUNICATIONS, EDS/SPM, 160 Bd Valmy, 92704 Colombes, France
}

\begin{abstract}
With new generation of Active Digital Radar Antenna, there is a renewal of waveform generation and processing approaches, and new strategies can be explored to optimize waveform design and waveform analysis and to benefit of all potential waveform diversity. Among these strategies, building and exploitation of the Noncircularity of waveforms is a promising issue. Up to the middle of the nineties, most of the signals encountered in practice are assumed to be second order (SO) circular (or proper), with a zero second correlation function. However, in numerous operational contexts such as in radio communications, the observed signals are either SO noncircular (or improper) or jointly SO noncircular with a particular signal to estimate, to detect or to demodulate, with some information contained in the second correlation function of the signals. Exploitation of this information in the processing of SO noncircular signals may generate dramatic gain in performance with respect to conventional processing and opens new perspective in signal processing. The purpose of this paper is to present a short overview of the interest of taking into account the potential SO noncircularity of the signals in signal processing and to describe the potential interest of SO noncircular waveforms for radar applications.

Index Terms - Noncircular, improper, widely linear, Single Antenna Interference Cancellation (SAIC), Binary signals, AM, ASK, BPSK, MSK, GMSK, demodulation, equalization, detection, beamforming, direction finding, MIMO, radar, waveform diversity.
\end{abstract}

\section{INTRODUCTION}

Up to the middle of the nineties, most of the signals encountered in practice are assumed to be SO circular [1], which means that their second correlation function is zero. Despite of the fact that many signals used in practical situations are indeed SO circular, some of them, mainly borrowed from the radio communications context, are SO noncircular [1] and contain information in their second correlation function. It is in particular the case for rectilinear signals such as Amplitude Modulated (AM), Amplitude Shift Keying (ASK) or Binary Phase Shift Keying (BPSK) signals [2]. It is also the case for signals which become quasirectilinear (i.e. a complex filtered version of a rectilinear signal) after a simple derotation operation such as Offset Quadrature Amplitude Modulations (OQAM), Minimum Shift Keying (MSK) or Gaussian MSK (GMSK) modulations [2]. Moreover, in the context of MIMO wireless systems [3] and whatever the chosen constellation, some Spatio-Temporal Block Coding (STBC) schemes, such as the Alamouti's scheme [4], generate observations which become jointly SO noncircular with the transmitted symbols. For observations which are either SO noncircular or jointly SO noncircular with a given input signal, exploitation of the information contained in the second correlation function of the signals may generate dramatic gain in performance with respect to conventional processing. This may even open new perspectives in signal processing.

The purpose of this paper is to present a brief overview of the interest of taking into account the potential SO noncircularity of the signals in signal processing and to describe the potential interest of noncircular waveforms in radar applications.

\section{SECOND ORDER NONCIRCULAR OBSERVATIONS}

The SO statistics of a $(N \times 1)$ complex random vector process $\boldsymbol{x}(t)$ are completely defined by the two correlation matrices $R_{x}(t, \tau) \triangleq \mathrm{E}\left[\boldsymbol{x}(t) \boldsymbol{x}(t-\tau)^{\dagger}\right]$ and $C_{x}(t, \tau) \triangleq \mathrm{E}[\boldsymbol{x}(t) \boldsymbol{x}(t-$ $\left.\tau)^{\mathrm{T}}\right]$, for all the values of $(t, \tau)$, where ${ }^{\dagger}$ and $\mathrm{T}$ are the transposition conjugation and the transposition operation respectively. The vector $\boldsymbol{x}(t)$ is said to be SO noncircular [1] if the pseudo-correlation matrix [5], $C_{x}(t, \tau)$, is not zero for at least one couple $(t, \tau)$. Such situations may for instance occur in the presence of rectilinear signals such as BPSK signals used in secondary radar or quasi-rectilinear signals such as GMSK signals used in the GSM network. Extension of the SO noncircularity concept to higher order may be found in [1] [6].

\section{SO NONCIRCULARITY EXPLOITATION THROUGH OTIMAL WIDELY LINEAR FILTERING IMPLEMENTATION}

\section{A. Widely linear filtering}

It is shown in [7] that for stationary observations, whose complex envelope is necessarily SO circular [1], SO optimal complex filters are linear and Time Invariant (TI), i.e. of the form $y(t) \triangleq \boldsymbol{w}^{\dagger} \boldsymbol{x}(t)$, where $\boldsymbol{w}$ is a TI complex filter. However, for a nonstationary observation, whose complex envelope may be SO noncircular, SO optimal complex filters are Time Variant (TV) and, under some conditions of SO noncircularity presented in [7] [8], Widely Linear (WL) [8], i.e. of the form $y(t) \triangleq \boldsymbol{w}_{1}(t){ }^{\dagger} \boldsymbol{x}(t)+\boldsymbol{w}_{2}(t) \boldsymbol{\dagger}^{\dagger}(t)^{*}$, where * means complex conjugate and where $\boldsymbol{w}_{1}(t)$ and $\boldsymbol{w}_{2}(t)$ are TV complex filters. For this reason, since the first use of WL filters in signal

Funded by French MoD (DGA/MRIS) 
processing [9] and the first works about their SO optimality in noncircular contexts [7] [8], there has been an increasing interest for such filters in signal processing. The main applications of WL filtering currently concern Co-Channel Interference (CCI) cancellation of noncircular interferences, equalization of frequency selective channels for noncircular transmissions and optimization of MIMO systems using either SO noncircular constellations or some particular "noncircular" STBC schemes.

\section{B. Cochannel Interference Rejection}

\section{1) Cellular F/TDMA radio communications systems}

SO optimal WL filters have been used for CCI rejection in F/TDMA radio communications cellular networks using either rectilinear modulations such as BPSK signals [10] or quasirectilinear modulations such as MSK [10] or GMSK [10-11] signals. In such contexts, it has been shown in [10] that SO optimal WL filters allow the processing of up to $P=2 N-1$ rectilinear or quasi-rectilinear interferences from an array of $N$ sensors. This proves in particular the capability of these filters to do Single Antenna Interference Cancellation (SAIC) $(P=1$ for $N=1)$. For this reason, the $3 \mathrm{G}$ Americas [12] presents the SAIC technology as a great improvement for GSM mobile station receivers, already standardized and allowing significant network's capacity gains for the GSM system. Figure 1 illustrates the behavior of both the conventional receiver and the optimal receiver, implementing the SAIC concept, when a BPSK useful signal is corrupted by a BPSK interference. Contrary to the conventional receiver, which compensates the phase of the useful signal before taking the real part of the observation, the SAIC receiver puts the interference on the y-axis before taking the real part of the observation, which eliminates the latter.

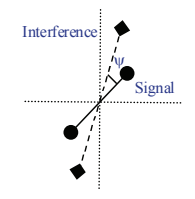

$x(k T)$

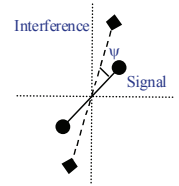

$x(k T)$

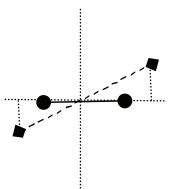

$y(k T)$

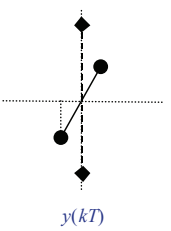

Figure 1 - SAIC concept for BPSK signal and interference

\section{2) CDMA radiocommunications systems}

SO optimal WL filters have been proposed to mitigate Multiple Acces Interference (MAI) in CDMA radio communications networks using either BPSK modulation [1316] or OQPSK modulation [17], through the implementation of WL Multi-user Detection (MUD) schemes. For Direct
Sequence Spread Spectrum (DSSS) BPSK signals, it is shown in [15-16] that the use of optimal WL filters for MUD allows to double the capacity of the system in terms of number of users for a given spreading factor. For OQPSK signals, which concern IS'95 and CDMA 2000 standard, the use of optimal WL filters strongly improves the performance of the receivers.

\section{3) Detection systems}

Very recently, optimal WL filters have been applied to the problem of detection of either a random signal [18] or a known deterministic signal with unknown parameters [19] corrupted by SO noncircular interferences. Several new GLRT-based detectors associated to several choices of unknown desired signal parameters have been developed in [19] for binary coded signals and arbitrary SO noncircular interferences. One of this new detector is also well-suited for synchronization purposes in radio communications [20]. All the proposed detectors allow to perform SAIC in the presence of a rectilinear interference as it is illustrated by figure 2 . The latter shows the variations of the Non Detection Probability of the desired BPSK signal at the output of several conventional (Ci) and optimal detectors (Nci) as a function of the input SNR in the presence of a BPSK interference such that INR = $\mathrm{SNR}+20 \mathrm{~dB}$, for a false alarm rate equal to 0.001 , for a signal containing 64 symbols and for $N=1$ sensor. Phase difference between the sources is equal to $45^{\circ}$. Note the non detection of the signal by the conventional receivers and the detection of the latter by the optimal receivers.

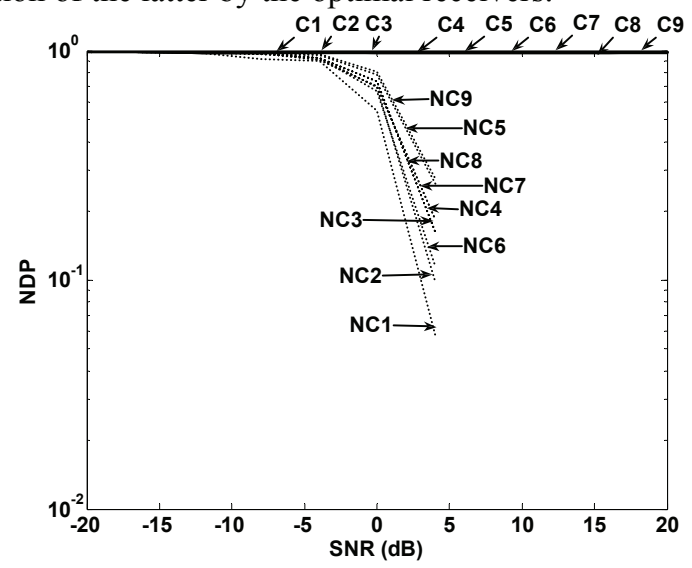

Figure 2 - Non detection probability as a function of SNR

\section{4) MVDR Beamforming for passive listening}

For passive listening applications, two WL MVDR beamformers have been proposed recently [21] to improve the performance of the well-known Capon's beamformer [22-23] in the presence of SO noncircular interferences. The first one is TI whereas the second one, TV, allows to process SO noncircular interferences having a nonzero frequency offset or carrier residue. The proposed TI MVDR beamformer allow the processing of up to $2(N-1)$ rectilinear interferences from an array of $N$ sensors.

\section{Egalisation of Linear Frequency Selective channels}




\section{1) Single carrier links}

In [24] several MMSE equalization schemes based on WL processing are proposed for rectilinear and quasi-rectilinear single carrier signals propagating through frequency selective channels, generating SO noncircular Inter-Symbol Interference (ISI) at the reception. It is shown in particular that WL equalizers outperforms Linear ones in such contexts.

\section{2) DMT or OFDM links}

Optimal Narrow-Band Interference (NBI) resistant WL receivers/equalizers have also been proposed in [25] and [26] for Discrete Multitone (DMT) and Orthogonal Frequency Division Multiplex (OFDM) systems using SO noncircular constellations. Reference [26] considers both Cyclic-Prefix (CP) and zero-padded based multicarrier (MC) systems whereas [25] limits to CP systems.

\section{3) UWB links}

In [27] and [28], WL equalizers are proposed to mitigate ISI in the context of direct-sequence based Ultra-Wideband (DS-UWB) systems using either BPSK [27] or 4BOK [28] modulations. The use of WL receivers allows very powerful high rate and short range transmission from the standardized UWB wave-forms under investigation for wireless personal area networks.

\section{MIMO systems optimization}

In [29], [30] and [31], WL equalizers are proposed to mitigate ISI in the context of MIMO systems using either BPSK constellation [29], [30] or STBC schemes, such as the Alamouti's scheme, which generate observation jointly SO noncircular with the transmitted symbols [31]. Strong gain in performance with respect to conventional linear equalizers are proved. In [32] and [33], WL receivers are proposed to mitigate interferences generated by some spatial multiplexing schemes involving either SO noncircular constellations [32] or transmitted symbols and their complex conjugates [33]. Finally reference [34] proposes optimized WL precoder schemes from rectilinear constellations and Channel state information knowledge at the transmitter.

\section{SO NONCIRCULARITY EXPLOITATION FOR PARAMETERS ESTIMATION}

The SO noncircularity of the signals may also be exploited for some parameter estimation problems without the implementation of WL filters. Such a property has been exploited for direction finding of rectilinear [35] [36] or SO noncircular signals [37], where up to $2 N-1$ or $2(N-1)$ rectilinear signals may be processed from an array of $N$ sensors. In [38] it is shown that for SO noncircular signals, blind channel identification is possible from SO statistics only. Finally several algorithms for blind frequency offset estimation of SO noncircular single carrier [39] [40] and OFDM [41] links through frequency selective channels have been proposed recently.

\section{NONCIRCULAR WAVEFORM EXPLOITATION FOR RADAR SYSTEMS}

Radar waveforms have been classically optimized relatively to ambiguity function for target detection (good range resolution; sufficient energy on target, non sensitivity to target Doppler offset, robustness to clutter and jamming,...). For the time being, Frequency Modulation waveforms are widely used and preferred to Phase Coding ones for Radar Applications. But noncircularity properties of phase coded radar waveforms have not yet been exploited, and this breakthrough coming from radiocommunication could modify balance between frequency \& phase coding choice.

From this matter of fact, we have to explore classical phase coded waveform : BPSK, Pseudo-Noise coded (PN) sequences, Multi-Phase codes, .... .

Waveforms based on Binary phase-coded sequences, like BPSK, are used in Radar but their noncircularity properties are not exploited. In radio communications, this Binary phasecoded sequences are exploited to transmit information in a multi-user environment or for discretion purposes (e.g. for spread-spectrum communication system using binary PSK modulation, M-sequences are applied as sub-baud codes for bandwidth expansion), but for radar applications, these codes are used in an other framework for "pulse compression" to increase range resolution. Furthermore in radar, target motion gives rise to a Doppler frequency offset on the received signals. It is thus necessary not only to analyse the coded waveforms auto-correlation functions, but also to study the ambiguity function $X(t, f)$ in time-frequency $(\mathrm{t}, \mathrm{f})$ plan, introduced by P. M. Woodward in 1953, to evaluate the performance under various Doppler frequency returns :

$X(t, f)=\int_{-\infty}^{+\infty} s(\tau) s^{*}(\tau+t) e^{-i 2 \pi f t} d \tau$

Radar Engineering will select $s(t)$ that will give an ambiguity function as close as possible to the ideal "thumbtack" response, with most of the weight around the origin in the $(\mathrm{t}, \mathrm{f})$ plane :

$|X(t, f)| \leq|X(0,0)|$

with

$$
\int_{-\infty}^{+\infty} \int_{-\infty}^{+\infty}|X(t, f)|^{2} d t d f=|X(0,0)|^{2}=1
$$

The ambiguity should still be small in some strip of the $(\mathrm{t}, \mathrm{f})$ plane containing the delay axis and because of the sidelobe on the Doppler axis, the strip must be narrower than $1 /(\mathrm{MT})$ in the Doppler direction.

Considering binary phase-coded sequences, like BPSK (with 0 and $\pi$ phase coding of the carrier waveform), Barker sequences have been introduced [42], as their aperiodic autocorrelations are all $1 / \mathrm{M}$ or 0 , for $\mathrm{k}=1,2, \ldots, \mathrm{M}-1$. However, while the ambiguity function is small on the delay axis, for all known Barker sequences there are substantial ridges away from the zero Doppler axis. Poor performance of Barker sequences is mainly due because they are all short sequences (there are no Barker sequences of length greater 
than 13, some elements of this proof in [43]), but exploitation of their non-circularity in anti-jamming or DOA could improve their advantages and other performances that could be attractive.

Pseudo-Noise coded (PN) sequences have been also studied as alternative of Barker sequences. The fact, that we have longer sequence could be also an advantage for noncircularity exploitation. They are generated by maximum length shift-register sequences, and have the property that the cyclic autocorrelation sidelobe levels are very small, equal to $1 / \mathrm{M}$ for $1 \leq \mathrm{k} \leq \mathrm{M}-1$. If we consider their aperiodic autocorrelations, their sidelobes are at most $\log (M) / \sqrt{M}$ (see [44]). The low level of cyclic autocorrelation Ambiguity function sidelobes $(-1 / \mathrm{M})$ only occurs for the zero Doppler case, while the remainder of the plot is similar to the aperiodic performance. The idea is that subsequences of long $\mathrm{M}$ sequences might be sufficiently random to have good autocorrelation properties, as studied in [46] where some properties of such subsequences (e.g. moments, mean weights) are calculated.

In practice, the ambiguity performance of binary phase coded sequences is lower than linear Frequency Modulation (FM) chirp signals. This is why Shift-register sequences have been deeply analyzed algebraically and investigated experimentally by simulation. For example, Bartee and Wood [49] have studied interlacing several short M-sequences having relatively prime lengths to obtain long codes having good short-term correlation properties to promote rapid acquisition in a radar ranging application. The general problem area involves the pseudo-randomness properties and/or weight distributions of segments from M-sequences. Most of the well-known good pseudo-random properties of $\mathrm{M}$-sequences pertain to averages over an entire period of the M-sequence. The detailed weight distribution of segments from M-sequences is not well known. Bartee and Wood [49] have considered an exhaustive comparison of all $\mathrm{M}$-sequences of lengths up to and including $M=2^{14}-1=16383$ for a wide range of subsequence lengths to select "optimum" Msequences. For a given subsequence length, M, and fixedlength M-sequences the "optimum" M-sequence, in the sense of Bartee and Wood [49], is essentially that one whose minimum weight M-tuple has greatest weight. In one of their examples, a particular-system performance parameter varies over more than five orders of magnitude from worst-case Msequence to best-case M-sequence. Unfortunately, Bartee and Wood found no systematic relationship between their set of optimum M-sequences and the corresponding generating functions.

Alternatively, Lindholm has considered the moments of the distribution of weights of M-tuples from any given Msequence, and develop a systematic relationship between the moments of the distribution and the characteristic polynomial via the notion of coset functions. In addition, the coset function approach yields a systematic relationship between the distributions of the entire family of M-sequences of any given length. The first few moments of a distribution are sufficient to describe the main geometrical features of the distribution.

As in communication, noncircularity could be exploited on Multi-Phase Codes. In Radar, the design of Multi-Phase codes has focused on small aperiodic autocorrelation, rather than maximum sidelobes minimization of the whole ambiguity function. For instance, Golomb [48] has developed a 6-phase analogues of Barker codes. But, as with Barker codes, the maximum Ambiguity function sidelobes of these codes, away from the delay axis, are high.

Performance of Phase coded sequences should be now considered in the new framework of noncircularity, as it is done in radio communications. As we have seen in the first part of the paper, we could accept degraded performance of ambiguity function if we exploit noncircularity to improve Radar detection, anti-jamming (twice more number of interferences sources that could be rejected, hostile interference or interferences from other co-localized sensors that share same frequency plan) and DOA (better resolution than MUSIC with circularity assumption) performances by new processing chains exploiting statistics contained in the complex symmetric unconjugated spatial covariance matrix, and not only the classical covariance matrix. A potential improvement could be obtained if the two covariance matrices are used simultaneously. Phase coded waveforms can be used also for data transmission (plots \& tracks) in netted radar system (in a dense network of co-localized radars, Phase coded waveforms could be used for communication modes between radars).

\section{CONCLUSION}

The area of waveform design enjoys a new attention due to advances in "software radar" concept (Digital Array, use of software in Front-End,...). Classically, radar waveforms have been designed to optimize ambiguity function for target detection properties (good range resolution; sufficient energy on target, non sensitivity to target Doppler offset, robustness to clutter and jamming,...). In practice, Frequency Modulation have been preferred to Phase Coding for Radar Applications. But for the time being, noncircularity properties of phase coded radar waveforms have not been exploited as it has been done in radio communications. We propose to study new phase coded waveforms and exploit statistics contained in the complex symmetric unconjugated spatial covariance matrix (pseudocovariance matrix), to improve radar performances (detection, anti-jamming, DOA,...) and to allow communication, with the same phase coded waveform, between co-localized radar sensors (information on targets plots/tracks are exchanged between radar sensors in a collaborative way).

In that respect, it highlights the importance of designing waveforms with diverse objectives in terms of global radar performances and not with only classical ambiguity function constraints.

Exploitation of radar waveforms noncircularity property could be also extended to be applied for multi-static, MIMO and Ultra-Wideband Radars applications. 
Concept of Waveform Diversity is a new challenging project that will promote new solutions and paradigms for advanced agile waveform resources management as explained in $[50]$.

As Woodward wrote in his seminal book, I could end with the following statement :

"The reader may feel some disappointment, not unshared by the writer, that the basic question of what to transmit remains substantially unanswered."

\section{REFERENCES}

[1] B. Picinbono, "On circularity", IEEE Trans. Signal Processing, Vol 42, $\mathrm{N}^{\circ} 12$, pp. 3473-3482, Dec. 1994.

[2] J.G. Proakis, "Digital communications", McGraw-Hill, 3dt Edition, 1995. [3] H. Boelcskei, D. Gesbert, C. Papadias, A. J. van der Veen (Editors) "Space-Time Wireless systems : From Array processing to MIMO communications", Cambridge Univ. Press ISBN 052185105x. Spring 2006.

[4] S.M. Alamouti, "A simple transmit diversity technique for wireless communications", IEEE Trans on Comm., Vol 16, pp. 1451-1458, Oct. 1998.

[5] F.D. Nesser, L. Massey, "Proper Complex random processes with applications to information theory", IEEE Trans. Information Theory, vol 39, No4, pp. 1293-1302, July 1993.

[6] P.O. Amblard, M. Gaeta, J.L. Lacoume, "Statistics for complex variables and signals - Part I and II", Signal Processing, Elsevier, Vol 53, $\mathrm{N}^{\circ} 1$, pp. 125, Aug. 1996.

[7] P. Chevalier, "Optimal array processing for non stationary signals", Proc. ICASSP, pp. 2868-2871, Atlanta (USA), May 1996.

[8] B. Picinbono, P. Chevalier, "Widely linear estimation with complex data", IEEE Trans. Signal Processing, Vol 43, №8, pp. 2030-2033, Aug. 1995.

[9] W.M. Brown, R.B. Crane, "Conjugate linear filtering", IEEE Trans. Information Theory, vol 15, N4, pp. 462-465, July. 1969

[10] P. Chevalier and F. Pipon, "New Insights into optimal widely linear array receivers for the demodulation of BPSK, MSK and GMSK signals corrupted by non circular interferences - Application to SAIC", IEEE Trans. Signal Processing, Vol 54, $\mathrm{N}^{\circ}$, March 2006.

[11] H. Trigui, D.T.M. Slock, "Performance bounds for cochannel interference cancellation within the current GSM standard", Signal Proccessing, Elsevier, Vol. 80, pp. 1335-1346, 2000

[12] M. Austin, "SAIC and synchronised networks for increased GSM capacity", 3 G Americas' SAIC Working group, Sept 2003.

[13] G. Gelli, L. Paura, A.R.P. Ragozini, "Blind widely linear multiuser detection", IEEE Communications Letters, vol 4, Nº6, pp. 187-189, June 2000.

[14] S. Buzzi, M. Lops, A.M. Tulino, "A new family of MMSE multiuser receivers for interference suppression in DS/CDMA systems employing BPSK modulation", IEEE Trans.on Com., Vol 49, Nº1, pp. 154-167, Jan. 2001.

[15] A.M. Tulino, S. Verdu, "Asymptotic analysis of improved linear receivers for BPSK-CDMA subject to fading", IEEE Journal. on Selected Areas in Communications, $\mathrm{Vol}$ 19, $\mathrm{N}^{\circ}$ 8, pp. 1544-1555, August 2001.

[16] S. Buzzi, M. Lops, "Performance analysis for the improved linear multiuser detectors in BPSK-modulated DS-CDMA systems", IEEE Trans.on Communications, Vol 51, $\mathrm{N}^{\circ} 1$, pp. 37-42, Jan. 2003

[17] A. Mirbagheri, K.N. Plataniotis, S. Pasupathy, "An enhanced widely linear CDMA receiver with OQPSK modulation", IEEE Trans.on Communications, Vol 54, N², pp. 261-272, Feb. 2006.

[18] P.J. Schreier, L.L. Scharf, C.T. Mullis, "Detection and Estimation of improper complex random signals", IEEE Trans on Info Theory, Vol 51, $\mathrm{N}^{\circ} 1$, pp. 306-312, Jan. 2005

[19] P. Chevalier, A. Blin, F. Pipon, F. Delaveau, "GLRT-Based array receivers to detect a known signal corrupted by noncircular interferences", Proc. EUSIPCO, Poznan (Poland), Sept. 2007.

[20] P. Chevalier, F. Pipon, F. Delaveau, "Second-order optimal array receivers for synchronization of BPSK, MSK and GMSK signals corrupted by noncircular interferences", Eurasip Journal on Advances in Signal Processing (JASP), Hindawi Publishing Corporation, Vol 2007, Article ID 45605, 16 pages, 2007.

[21] P. Chevalier, A. Blin, "Widely linear MVDR beamformer for the reception of an unknown signal corrupted by noncircular interferences", IEEE Trans. Signal Processing, Vol 55, N11, pp. 5323-5336, November 2007.
[22] J. Capon, "High resolution frequency wave number spectrum analysis", Proc. IEEE, vol 57, No8, pp. 1408-1418, Aug. 1969.

[23] J. Capon, R.J. Greenfield, R.J. Kolker, "Multidimensional maximum likelihood processing of a large aperture seismic array", Proc. IEEE, vol 55, No2, pp. 191-211, Feb. 1967.

[24] W.H. Gerstacker, R. Schober, A. Lampe, "Receivers with widely linear processing for frequency-selective channels", IEEE Trans. On Communications, Vol 51, Nº, pp. 1512-1523, Sept. 2003.

[25] D. Darsena, G. Gelli, L. Paura, F. Verde, "Widely linear equalization and blind channel identification for interference-contaminated multicarrier systems", IEEE Trans. Sign. Proc, Vol 53, No3, pp. 1163-1177, March 2005. [26] D. Darsena, G. Gelli, F. Verde, "Universal Linear Precoding for NBIproof Widely Linear Equalization in MC systems", Eurasip Journal on Wireless Communications and Networking, Hindawi Publishing Corporation, Vol 2008, Article ID 321450, 13 pages, 2008.

[27] A. Parihar, L. Lampe, R. Schober, C. Leung, "Equalization for DSUWB Systems - Part I: BPSK modulation", IEEE Trans. On Communications, Vol 55, Nº6, pp. 1164-1173, June 2007.

[28] A. Parihar, L. Lampe, R. Schober, C. Leung, "Equalization for DSUWB Systems - Part II : 4BOK modulation", IEEE Trans. On Communications, Vol 55, Nº, pp. 1525-1535, Aug 2007.

[29] D. Mattera, L. Paura, F. Sterle, "Widely Linear MMSE equaliser for MIMO linear time-dispersive channel", Electronics Letters, Vol 39, No20, pp. 1481-1482, Oct 2003.

[30] D. Mattera, L. Paura, F. Sterle, "Widely Linear Decision-Feedback Equalizer for Time-Dispersive linear MIMO channels", IEEE Trans. Signal Processing, Vol 53, No7, pp. 2525-2536, July 2005.

[31] W.H. Gerstacker, F. Obernosterer, R. Schober, A.T. Lehmann, A. Lampe, P. Gunreben, "Equalization concepts for Alamouti's space-time block code", IEEE Trans. On Communications, Vol 52, Nº, pp. 1178-1190, July 2004.

[32] S. Buzzi, M. Lops, S. Sardellitti, "Widely linear reception strategies for layered space-time wireless communications", IEEE Trans.on Signal Proc., Vol 54, N6, pp. 2252-2262, Jan. 2006.

[33] M. Witzke, "Linear and Widely linear filtering applied to iterative detection of generalized MIMO signals", Annales Télécommunications, Vol 60, $\mathrm{N}^{\circ} 1-2$, pp. 147-168, 2005.

[34] F. Sterle, "Widely linear MMSE transceivers for MIMO channels", IEEE Trans.on Signal Proc., Vol 55, N8, pp. 4258-4270, Aug. 2007.

[35] P. Gounon, C. Adnet, J. Galy, "Localisation angulaire de signaux non circulaires", Traitement du Signal, Vol 15, №1, pp. 17-23, Jan. 1998

[36] P. Charge, Y. Wang, J. Saillard, "A non-circular source direction finding method using polynomial rooting", Signal Processing, Vol 81, pp. 1765-1770, 2001.

[37] H. Abeida, J.P. Delmas, "MUSIC-like Estimation of Direction of Arrival for Noncircular Sources", IEEE Trans. Signal Processing, Vol 54, $\mathrm{N}^{\circ} 7$, pp. 2678-2690, Jul 2006.

[38] O. Grellier, P. Comon, B. Mourrain, P. Trebuchet, "Analytical blind channel identification", IEEE Trans. Signal Processing, Vol 50, №9, pp. 2196-2207, Sept 2002.

[39] P. Ciblat, P. Loubaton, E. Serpedin, G. Giannakis, "Performance analysis of blind carrier frequency offset estimators for non circular transmissions through frequency-selective channels", IEEE Trans. Sig; Proc; Vol 50, ํ4, pp. 130-140, Jan 2002

[40] A. Napolitatno, M. Tanda, "Doppler-Channel Blind Identification for non-circular transmissions in multiple access systems", IEEE Trans. on Communications, Vol 52, №12, pp. 2073-2078, Dec. 2004.

[41] T. Fusco, M. Tanda, "ML-based symbol timing and frequency offset estimation for OFDM systems with noncircular transmissions", IEEE Trans. on Signal Processing, Vol 54, Nº, pp. 3527-3541, Sep. 2006.

[42] R.H. Barker, "Group synchronisation of binary digital systems", pp. 273287 , in JACKSON, W. (Ed.): 'Communications theory', Academic Pres, New York, 1953

[43] M.L. Fredman \& al "Polynomes reciproques: conjecture d'Erdos en norme $\mathrm{L}^{4}$, taille des autocorrelalions et inexistence des codes de Barker ». C. R. Acad. Sci., Paris, vol. 308, Serie 1, pp. 461-464, 1989

[44] R.J. McEliece, "Correlation properties of sets of sequences derived from irreducible cyclic codes", Inf. \& Control, n 45 , pp. 18-25, 1980

[45] R. Skaug, "Numerical evaluation of the nonperiodic autocorrelalion parameter for optimal phases of maximal length sequences", IEE Proc. F, Commun., Radar and Signal Process., 127, (3), pp. 230-237, 1980

[46] J.H. Lindholm, "An analysis of the pseudo-randomness properties of subsequences of long m-sequences", IEEE Trans., IT-14, pp. 569-576, 1968 
[47] M.B. Pursley, and H.F.A. Roefs, "Numerical evaluation of correlation parameters for optimal phases of binary shit register sequences', IEEE Trans., COM-27, (10), pp. 1597-1604, 1979

[48] S.W. Golomb and R.A. Scholtz, R.A., "Generalised Barker sequences", IEEE Trans., IT-11,p p. 533-537, 1965

[49] T.C. Bartee and P.W. Wood, "Coding for tracking Radar ranging”, MIT Lincoln Lab, Lexington, Mass., Tech. Rep. 318, June 11, 1963

[50] F. Barbaresco, "New Agile Waveforms Based on Mathematics \& Resources Management of Waveform Diversity", NATO Lecture, SET-119, September 2008 